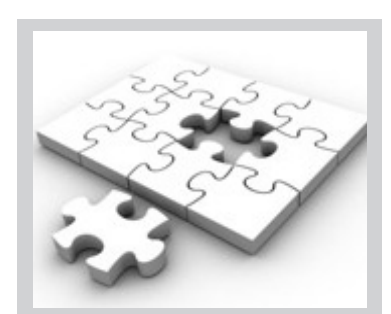

In an effort to facilitate the selection of appropriate peer reviewers for the African Journal of Primary Health Care \& Family Medicine, we ask that you take a moment to update your electronic portfolio on https://phcfm.org for our files, allowing us better access to your areas of interest and expertise, in order to match reviewers with submitted manuscripts.

If you would like to become a reviewer, please visit the journal website and register as a reviewer.

To access your details on the website, you will need to follow these steps:

1. Log into the online journal at https:// phcfm.org

2. In your 'user home' [https://phcfm.org/index. $\mathrm{php} / \mathrm{phcfm} / \mathrm{user}$ ] select 'edit my profile' under the heading 'my account' and insert all relevant details, bio statement and reviewing interest(s).

3. It is good practice as a reviewer to update your personal details regularly to ensure contact with you throughout your professional term as reviewer to the African Journal of Primary Health Care \& Family Medicine.

Please do not hesitate to contact us if you require assistance in performing this task.

Publisher: publishing@aosis.co.za Tel: +27 219752602

Tel: 0861000381

\title{
Acknowledgement to reviewers
}

The editorial team of the African Journal of Primary Health Care \& Family Medicine recognises the value and importance of the peer reviewer in the overall publication process - not only in shaping the individual manuscript, but also in shaping the credibility and reputation of our journal.

We are committed to the timely publication of all original, innovative contributions submitted for publication. As such, the identification and selection of reviewers who have expertise and interest in the topics appropriate to each manuscript are essential elements in ensuring a timely, productive peer review process.

We would like to take this opportunity to thank all reviewers who participated in shaping this volume of the African Journal of Primary Health Care \& Family Medicine. We appreciate the time taken to perform your review(s) successfully.

Abayomi O. Ayodapo

Abdul K.M. Hamdulay

Alan J. Barnard

Alida S. du Plessis-Faurie

Andrew J. Ross

Andrew L. Gray

Angeni Bheekie

Anne-Marie Bergh

Anthony C. Smith

Arvin Bhana

Aviwe P. Mgobozi

Bernhard M. Gaede

Billy M. Tsima

Bob Pattinson

Bongi Sibanda

Brenda Tusubira

Carine Prinsloo

Catherine O. Egbe

Catriona I. Macleod

Christine Rogers

Claire van Deventer

Claire L. Barrett

Colleen Aldous

Cristina Lopez del Burgo

David R. Edwards

David A. Cameron

Debbi Marais

Denon Tshienda

Detlef R. Prozesky

Dieudonné Mpunga-Mukendi

Dirk J. Blom

Don O'Mahony

Doreen K.M. Kaura

Doudou K. Nzaumvila

Edward Kunonga

Elizabeth M. Webb

Elizabeth Mason

Elma de Vries

Eric H. Decloedt

Esmarie van Tonder

Esther O. Oluwole

Farai D. Madzimbamuto

Febisola I. Ajudua

Françoise Guigné

George J. Hofmeyr

Gert Marincowitz

Gina Joubert

Godfrey Woelk

Gouwa Dawood

Grant Murewanhema
Hanneke Brits

Hassan Mahomed

Heike I. Geduld

Henry Lawson

Ian D. Couper

Ibrahim S. Bello

Inam Haq

Inam Chitsike

Ineke Buskens

Jagidesa Moodley

Jakobus M. Louw

James D. Porter

Jana Muller

Jane McCartney

Jeremiah Laktabai

Joel A. Dave

Johannes (Jannie) F. Hugo

Juan M. Jansen van Vuuren

Kantharuben Naidoo

Karin Joubert

Kate Rees

Kathryn Spangenberg

Kekeli K. Adanu

Keneilwe Motlhatlhedi

Keneni B. Daka

Kenneth Yakubu

Klaus B. von Pressentin

Kwabena A. Kyei

Laetitia Nyirazinyoye

Lana Avramenko

Langalibalele $\mathrm{H}$. Mabuza

Lauren Hutton

Lenon Gwaunza

Leslie London

Louis S. Jenkins

Margaret Williams

Marietjie R. de Villiers

Marlon-Ralph Nyakabau

Masemiano P. Chege (deceased)

Maylene Shung-King

Mea van Huyssteen

Megan Mahoney

Memory Munodawafa

Mergan Naidoo

Momin R. Butt

Munashe L. Nyika

Munyaradzi Madhombiro

Musa Dankyau

Naimah Ebrahim Khan

Nana K. Ayisi-Boateng 
Natasha Blanckenberg

Nelisiwe Khuzwayo

Nokuthula G. Nkosi-Mafutha

Oliver Johnson

Olufemi B. Omole

Olufemi O. Desalu

Olukayode A. Adeleke

Owen O. Eales

Parimalaranie Yogeswaran

Paul Rheeder

Peter C. Clarke-Farr

Pius O. Ameh

Portia J. Jordan

Priscilla Mataure

Pugie T. Chimberengwa

Radiance M. Ogundipe

Rafiat A. Anokwuru

Ramprakash Kaswa

Ran van der Wal

Rita Shomba

Roger N. Mabiala

Roy Mubuuke Gonzaga

Sa'ad Lahri

Sanele Ngcobo

Sara van Velze

Sebaka Malope

Serge Ngaima

Seter Siziya

Shabir Moosa

Solome K. Bakeera
Stan Houston

Stanley Tapesana

Star Khoza

Stefan Jansen van Vuuren

Stephen P. Merry

Steve G. Swartz

Steve Reid

Sunanda C. Ray

Susan A. Nzenze

Tasleem Ras

Tessa S. Marcus

Thandaza C. Nkabinde

Thandi Puoane

Tijani I.A. Oseni

Tinashe Goronga

T.K. Sundari Ravindran

Toby Helliwell

Tombo Bongongo

Tonya M. Esterhuizen

Ts'epo Motsohi

Tuviah Zabow

Vanessa Lomas-Marais

Vincent K. Cubaka

Violet K. Moselakgomo

Werner Viljoen

Wilhelm J. Steinberg

William B. Ventres

Witness Mudzi

Xikombiso G. Mbhenyane

Zelra Malan 